\title{
Protective efficacy of a genetic subunit bacterin against edema disease of swine in mice
}

\author{
X.L. Yang ${ }^{1,2 *}$, Y.J. Liu ${ }^{3 *}$, L.Q. Hu ${ }^{1,2}$, L.W. Guo ${ }^{1,2}$, G.P. Liu' ${ }^{1,2,4}$, B. $\mathrm{Wu}^{2,4}$ and \\ H.C. Chen ${ }^{2,4}$ \\ ${ }^{1}$ College of Animal Science, Yangtze University, JingZhou, HuBei, China \\ ${ }^{2}$ Cooperative Innovation Center for Sustainable Pig Production, Wuhan, China \\ ${ }^{3}$ Center for Animal Disease Control and Prevention of China, Beijing, China \\ ${ }^{4}$ State Key Laboratory of Microbiology, Huazhong Agricultural University, \\ WuHan, HuBei, China \\ *These authors contributed equally to this study. \\ Corresponding author: G.P. Liu \\ E-mail: Liuguoping001@yeah.net
}

Genet. Mol. Res. 14 (4): 18026-18033 (2015)

Received August 20, 2015

Accepted October 8, 2015

Published December 22, 2015

DOI http://dx.doi.org/10.4238/2015.December.22.29

ABSTRACT. The exotoxin SLT-IleB from the Escherichia coli Ee strain was expressed in E. coli, and the recombinant protein was purified, mixed with the Ee strain, then emulsified with oil-emulsion adjuvants to obtain a mixed subunit bacterin. Groups of Kunming mice were immunized at weeks 0 and 2, and challenged intraperitoneally with the Ee strain at week 4. Antibodies were detected by ELISA and an agglutination test. After the second immunization, the antibody level increased and the rate of immune protection against the Ee strain was 70 and $91.7 \%$ in the subunit bacterin and bacterin groups, respectively. Therefore, the mixed subunit bacterin provided good protection against the homologous Ee strain, which provides a basis for further research, 
into high-efficacy vaccines against porcine edema disease.

Key words: Expressed exotoxin; Subunit bacterin; Mice; Protection efficacy

\section{INTRODUCTION}

Swine edema disease is common in weaning piglets and is induced by a serotype of Shigella like toxin Escherichia coli (SLTEC). This is an important disease because it negatively affects scale aquaculture (Linggood and Thompson, 1987; Karch and Meyer, 1989; Karch et al., 1995; Ludwig et al., 1996). SLT-Ile is the major toxin of SLTEC, which induces edema disease in piglets. During swine edema disease, pathogenic $E$. coli attach to epithelial cells within the small intestine using F18ab (F107) fimbriae. SLT-Ile, produced by resident and progenitor bacteria in the gut, is absorbed through the intestinal cells. SLT-Ile can specifically bind to the Gb4 receptor on intestinal epithelial cells through subunit $B$, and mediate subunit $A$ into cellular mitochondria. Subunit A has $\mathrm{N}$-glycosidase activity, which can specifically break down the glycosidic bond of the adenine located at position 4324 of the 5 '-end of $28 \mathrm{~S}$ rRNA. The unlinked adenine residues then prevent binding between amino-acylation tRNA on elongation factor EF-1 and the 60S mitochondrial subunit, and subsequently interrupt protein synthesis, which induces metabolic dysfunction and cell death (DeGrandis et al., 1987; Imberechts et al., 1992; Bertschinger et al., 2000).

To prevent and treat edema disease, it is necessary to reduce the amount of pathogenic E. coli inside the intestinal track. However, the appearance of drug-resistant strains resulting from the overuse of antibiotics has made vaccination a preferred way to prevent this disease. With increasing studies being performed on the pathogenic mechanism of swine edema disease, researchers have suggested that the ideal vaccine would work by preventing $E$. coli attachment in the intestinal epithelium in piglets, thus, protecting piglets against the attachment of SLT-Ile. Vaccines based on inactivated strains are currently used in China to prevent edema disease, due to the unstable effect of the $\mathrm{O}$ antigen on the immune system. On the other hand, researchers in other countries have used conteracting toxic substances following active immunization with purified SLT-Ile toxin and passive immunization using SLT-Ile hyper-immune serum, which both result in significant immune effects (Garrity et al., 1997; Johansen et al., 1997). These data suggest that it is necessary to develop corresponding toxic factors as alternatives to antigens for the prevention of edema disease. The traditional method of extracting toxins from supernatants derived from $E$. coli culture is costly, and separation and purification of toxins is difficulty due to the small molecular weight of subunit $B$, which is unsuitable for large scale production. Thus, we should consider the protection provided by both strain and toxin by preparing a subunit vaccine using a recombinant protein and an inactive strain and investigating the effect of a subunit vaccine on mice in order to lay the foundation for developing new types of vaccines against $E$. coli in swine edema disease.

\section{MATERIAL AND METHODS}

\section{Bacterial strain}

The Ee edema disease coliform of swine was extracted and conserved in our lab. The 
construction of BL21 (DE3) containing three copies of SLT-IleB was also supported by our lab.

\section{Primary reagents}

FIA and SKL were from Sigma Ltd.; IPTG was from Promega; HRPO IgG was from Sout hemBiotech; lysozyme, and oxidized and reduced forms of glutathione were from Yuan Pinghao Ltd., Beijing.

\section{Experimental animals}

Female Kunming rats with six yearlings were purchased from Hubei Academy of Medical Sciences. In total 56 rats were randomly separated into four groups, with 14 rices in each group. Four mice were selected and used to detect antibody level. The remaining 10 animals were used in the counteracting toxic substances test.

\section{Cultivation and bacterial count}

The procedure was performed in accordance with the routine experimental method used in molecular laboratories.

\section{Expression and purification of 3 copy of SLT-IleB}

The BL21 bacterial strain containing the target plasmid was vaccinated and cultivated overnight. The next day, samples were vaccinated into a large flask at a 1:100 concentration. When the exponential phase was reached at $37^{\circ} \mathrm{C}\left(\mathrm{OD}_{600}=0.6-1.0\right)$, IPTG was added at $1 \mathrm{mM}$ for $3 \mathrm{~h}$. Bacteria were induced by heating with a high frequency ultrasonic wave. After centrifugation, the inclusion bodies in the sediment were dissolved in $0.6 \%$ SKL TE. Subsequently, bacteria were cultured under normal conditions for $0.5-2 \mathrm{~h}$ and were then centrifuged. Next, the liquid supernatant was dialyzed and renatured, and the sample was subjected to SDS-PAGE. Coomassie brilliant blue (R250) was used to determine the purity of the inclusion body. Protein concentration was determined using an ultraviolet spectrophotometer.

\section{Vaccine preparation}

The cultivation of the Bacteria vaccine seeds Ee was performed following the routine procedure. After centrifugation, formaldehyde phosphate buffer was added to inactivate the bacteria. Subsequently, the inactive Ee bacteria strain was mixed with certain proportions of inactivated bacteria and the expressed protein. The emulsified and prepared bacteria are referred to as the subunit bacterial vaccine (containing 1 billion Ee per $\mathrm{mL}$, and $\sim 100 \mu \mathrm{g}$ of the edema toxin). The inactive Ee bacterial strain was prepared and the cell concentration was maintained at the same level as the subunit bacterial vaccine. A mixture of the subunit bacterial vaccine and the unit bacterial vaccine was injected into the rat and repeated every 2 weeks. Animals in the control group were injected with an equivalent volume of PBS. After the first injection, blood was collected every 2 weeks. At each collection, an equal amount of blood was used to perform an indirect ELISA and an agglutination of thallium test to detect the average toxin antibody level and the average bacteria agglutination titer, respectively. 


\section{Antibody detection}

Antibody detection was performed following the encyclopedia and the ELISA method was used. The detection of Ee was based on the trivial agglutination test.

\section{Determination of toxin levels}

To determine the $\mathrm{LD}_{50}$ of the Ee strain, $1.3 \times 10^{9} \mathrm{CFU}\left(5 \times \mathrm{LD}_{50}\right)$ of the toxin was injected into the rats. After 15 days, we observed and recorded the clinical features of the experimental rats.

\section{RESULTS}

\section{Extraction of toxin inclusion body}

A routine method was used to extract the inclusion body. Following denaturation and renaturation, the purity of the sample was determined by SDS-PAGE. In Figure 1, the lines with arrows are the three copy SLT-IleB fusion proteins with a size of $56 \mathrm{kDa}$.
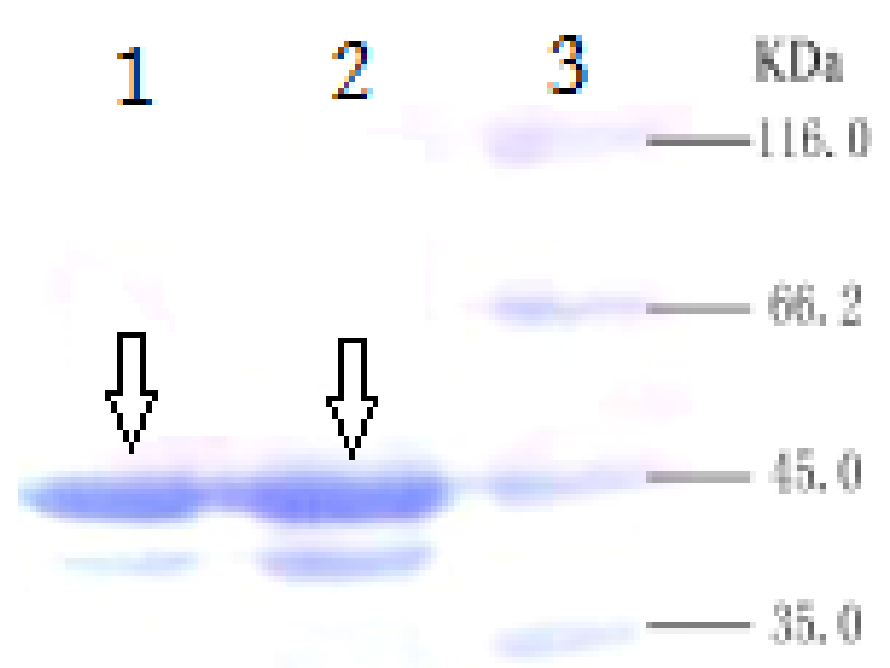

Figure 1. SDS-PAGE results of the expressed SLT-IleB inclusion body. 


\section{Changes in the antibody concentration following two immunizations}

Tail blood was collected two weeks after immunization and was used to determine the agglutination titer of ELISA antibody and the thallium from the SLT-IleB. Antibodies were detected in blood taken from the subunit vaccine group but not in the inactive vaccine group. The agglutination titer of bacterial antibody was 1:4 for the two groups. Two weeks after the second immunization, the average antibody levels as determined by ELISA in the subunit vaccine group were the highest, and the average antibody levels determined by of ELISA in the inactive vaccine group were also high but followed a declining trend. The agglutination titer of the bacterial antibody was 1:32 for the two groups, details relating to the first and second immunizations are shown in Figures 2 and 3.

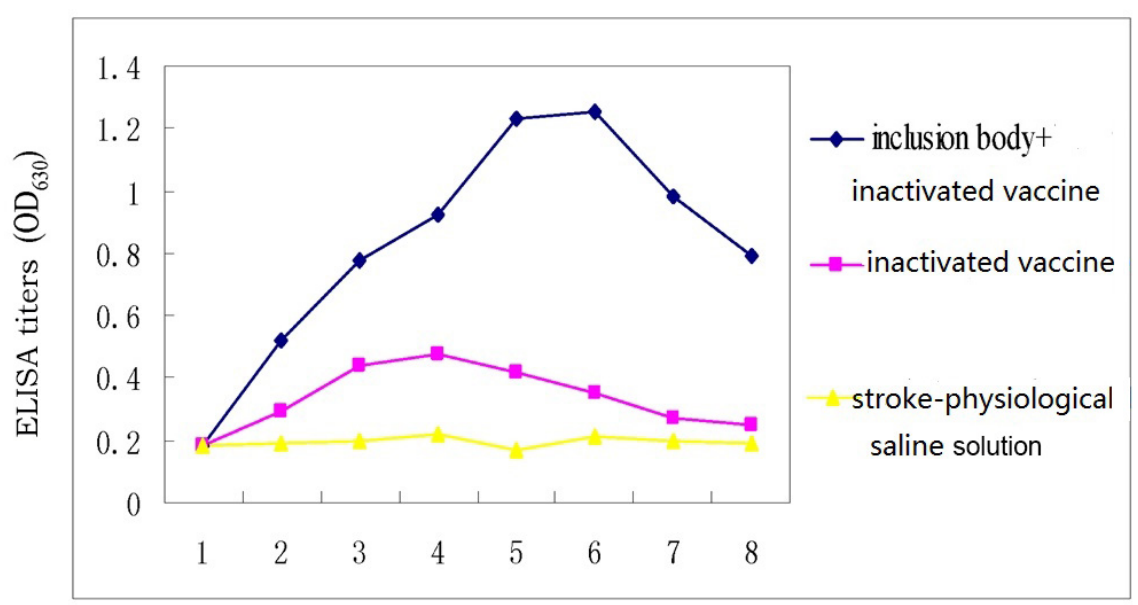

Days post-vaccination (weeks)

Figure 2. ELISA IgG titers against SLT-IleB after first immunization (mean $\mathrm{OD}_{630}$ ).

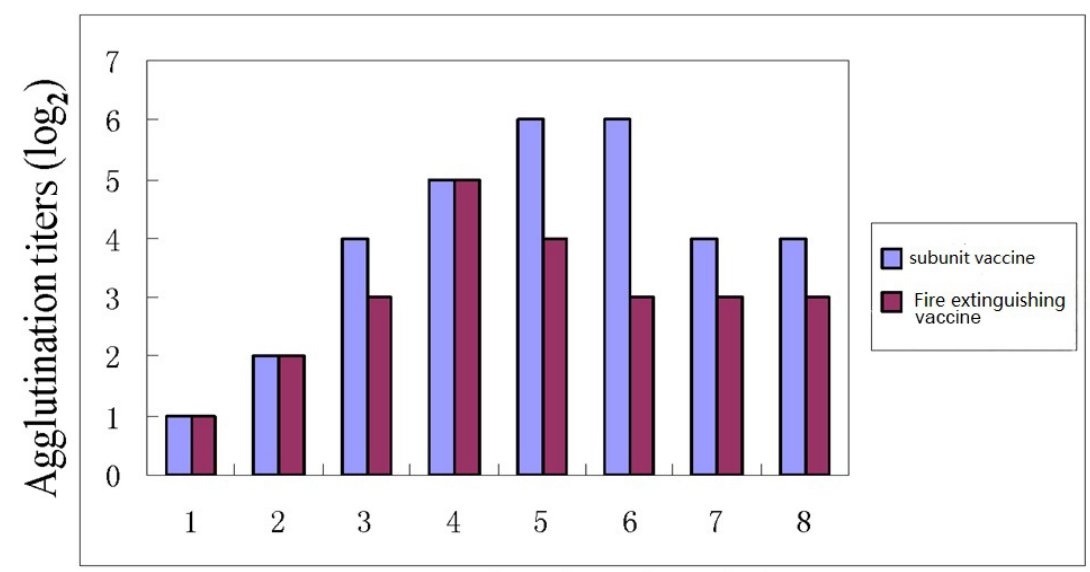

Days post-vaccination (weeks)

Figure 3. Agglutination titers after first immunization. 
The average antibody levels detected by ELISA, and the agglutination titer of the subunit and inactive vaccine groups, increased dramatically and were maintained at a high level after the second immunization. The average antibody levels in the inactive vaccine group as determined by ELISA increased slightly but remained relatively low (Figure 2). Furthermore, the peak agglutination titer obtained from the subunit vaccine group was higher than that obtained for the inactive vaccine group (Figure 3).

\section{Result of counteracting toxic substances}

Two weeks after the second immunization, the 5LD50Ee strain was adopted to perform the counteracting toxic substances test in both the case and control groups. All rats in the control group died within $12 \mathrm{~h}$. All animals were observed to have eyelid and facial edema, accompanying ataxia, paralysis or convulsions, and other neural symptoms within 4-12 h of receiving the injection. Autopsy revealed bleeding in the lungs, edema in the mesenteric serous and the stomach wall, and edema and bleeding in the intestines. Infectious bacteria were recovered from the heart, liver, spleen, and cerebrospinal fluid. In the study group, only one rat died $6 \mathrm{~h}$ after receiving the injection in the subunit vaccine group, and the surviving animals were subdued, not eating, and flocking together before they gradually returned to normal. These animals had returned to their pre-injection status by day 3 ; thus, the rate of protection was $90 \%$ (9/10 animals). Three mice died within $6-12 \mathrm{~h}$ post-injection in the inactive vaccine group, and the surviving rats were all subdued, noneating, and flocking together before they gradually returned to normal. All surviving animals had returned to their pre-injection status by day 3 ; thus, the rate of protection was $70 \%$ (7/10 animals).

\section{DISCUSSION}

Swine edema disease is an endemic or sporadically occurring disease that is a critical problem affecting swine production in China and throughout the world. During infection, morbidity can reach $30-40 \%$ with a mortality rate of $80-100 \%$ among infected individuals. Swine edema disease is usually observed in piglets 1-2 weeks after weaning, especially in piglets with high growth performance, leading to substantial economic loss. In addition to vaccines, other alternatives exist that can be used to prevent the negative effects of this disease. However, these alternatives are inefficient, which makes the development of highly efficient vaccines the primary choice for preventing swine edema disease.

More than $10 \mathrm{E}$. coli serotypes have been observed in swine edema disease, which makes it harder to prevent. Furthermore, it is clear that the use of a traditional inactivated vaccine is not sufficient to prevent the disease. The pathogenic factors of $E$. coli that are involved in edema disease include SLT-Ile and F18, and edema toxin SLT-Ile is the main factor that leads to edema. The subunit SLT-IleB, which does not possess enzymatic activity, is a protective antigen against the edema toxin, and it has an important role in cross protection between serotypes. Therefore, new types of vaccines that involve both the bacterium and the toxin are likely to be highly efficient in the prevention swine edema disease.

The Ee strain of E. coli from swine edema disease was isolated in our lab from a local bacterial strain found in Wuhan. Studies in mice have shown this strain to have high pathogenicity. A bacteria-free filtrate obtained from Ee strain broth culture can lead to death in mice, and organ edema is observed at autopsy. In addition, this filtrate leads to the death of vero cells in vitro. 
Typical symptoms of edema disease are observed in weaning pigs following intravenous injection of the Ee strain. Subunit B of the edema toxin has a low molecular weight making it difficult to separate and purify, and unsuitable for large-scale production. However, this can be overcome by the use of genetic engineering. According to previous studies, the combination and expression of copy 3 SLT-IleB enhances the biological activity and immunogenicity of the expressed protein. Thus, we choose to produce copy 3 SLT-IleB as a candidate toxin.

Antibody levels of the edema toxin B subunit in the subunit strain group were significantly higher than in the normal inactivated strain group. This shows that the addition of recombinant proteins to vaccines confers biological activity and good immunogenicity. Interestingly, the trend observed for agglutination titer in the subunit strain group was similar to that observed in the control group, whereas the agglutination titer was higher. The mechanism responsible for this difference is not clear. It is possible that this response was to the presence of the edema toxin receptor on vascular endothelial cells. Recombination of SLT-IleB stimulated these receptors, and the subsequent response of the organism against the vaccine strain; therefore, it is unclear whether SLT-IleB acts as an antigen or as an antigen and an adjuvant.

The preliminary results described here show that the subunit strain provides high protection $(90 \%)$ against the attraction by Ee strain which proved our expressive toxin may be the alternative of natural toxin to enhance normal inactivated strain's protection for virulent strain. Nonetheless, neither the inactivated strain nor the subunit vaccine strain was able to fully protect against the disease, which may have been due to the experimental design of the study. The purpose of our trial was to investigate the feasibility of using a subunit vaccine strain, in other words, to investigate whether recombinant proteins would significantly increase the protective ability of a vaccine and to determine whether protein recombinants can be used to replace natural toxins. Therefore, we choose to use a relatively low bacterial concentration when preparing the vaccine. These data suggest that recombinant proteins may be used as alternatives additives to vaccines, and that subunit strain vaccines are feasible in practice.

In conclusion, advantages of using a subunit strain vaccine comprising an inactivated vaccine and a recombinant subunit produced via genetic engineering are shown. This approach is feasible in practice and could lay the foundation of further research into new and efficient $E$. coli vaccines for use to prevent swine edema disease.

\section{Conflicts of interest}

The authors declare no conflict of interest.

\section{ACKNOWLEDGMENTS}

Research supported by the Special Fund for Agro-Scientific Research in the Public Interest (\#201503226).

\section{REFERENCES}

Bertschinger HU, Nief V and Tschäpe H (2000). Active oral immunization of suckling piglets to prevent colonization after weaning by enterotoxigenic Escherichia coli with fimbriae F18. Vet. Microbiol. 71: 255-267.

DeGrandis S, Law H, Brunton J, Gyles C, et al. (1989). Globotetraosylceramide is recognized by the pig edema disease toxin. J. Biol. Chem. 264: 12520-12525. 
Garrity RR, Rimmelzwaan G, Minassian A, Tsai WP, et al. (1997). Refocusing neutralizing antibody response by targeted dampening of an immunodominant epitope. J. Immunol. 159: 279-289.

Imberechts H, De Greve H and Lintermans P (1992). The pathogenesis of edema disease in pigs. A review. Vet. Microbiol. 31: 221-233.

Johansen M, Andresen LO, Jorsal SE, Thomsen LK, et al. (1997). Prevention of edema disease in pigs by vaccination with verotoxin 2e toxoid. Can. J. Vet. Res. 61: 280-285.

Karch $\mathrm{H}$ and Meyer $\mathrm{T}$ (1989). Single primer pair for amplifying segments of distinct Shiga-like-toxin genes by polymerase chain reaction. J. Clin. Microbiol. 27: 2751-2757.

Karch H, Rüssmann H, Schmidt H, Schwarzkopf A, et al. (1995). Long-term shedding and clonal turnover of enterohemorrhagic Escherichia coli 0157 in diarrheal diseases. J. Clin. Microbiol. 33: 1602-1605.

Lin YY (2006). Preliminary study on ELISA method for escherichia coli antibody detection and genetic subunit vaccine against porcine edema disease. HuaZhong Agriculture University.

Linggood MA and Thompson JM (1987). Verotoxin production among porcine strains of Escherichia coli. J. Med. Microbiol. 24: 359-362.

Liu GP, Wu B, Liu MY, He QG, et al. (2005). Detection, identification and biological characterization of Escherichia coli eliciting edema of piglet. Chin. J. Vet. Sci. 25: 31-33.

Ludwig K, Bitzan M, Zimmermann S, Kloth M, et al. (1996). Immune response to non-2O157 Vero toxin2 producing Escherichia coli in patients with hemolyticuremic syndrome. J. Infect. Dis. 174: 1028-1039.

Wu WF, Wu YS, Li JA, Lai YH, et al. (2002). Detecting chicken anti swine Escherichia coli antibody method explore using micro agglutination test. Guangdong J. Anim. Vet. Sci. 1: 33-35. 